\title{
A MODEL OF THE DISTRIBUTION OF IONISED CARBON IN M17SW.
}

\author{
Simon Pinnock and Tania S. Monteiro. \\ Queen Mary \& Westfield College, \\ Mile End Road, \\ London, E1 $4 N S$.
}

\begin{abstract}
The observed distribution of ionised carbon with depth into the edge-on photodissociation region (PDR) in M17SW is not consistent with results from chemical models which assume a constant or smoothly varying density. These observations have been interpreted to indicate that the PDR actually consists of many clumps embedded in a low density interclump medium (1). A chemical model is described which is designed to simulate such a clumpy PDR.
\end{abstract}

\section{Introduction.}

When a high mass star forms inside a molecular cloud it ionises the material around it to form an HII region. Between this and the cool molecular cloud exists a warm layer consisting of material which is partly ionised and photodissociated by far ultraviolet radiation (FUV) from the star. These photodissociation regions (PDR) are of great interest since they may be places where star formation is induced by the interaction of previously formed young stars with their parent molecular cloud. To interpret observations of these systems in terms of their physical characteristics, chemical models are used to predict the abundance, and to varying degrees the excitation of the observed species. When applied to observations of an edge-on system like the HII region/molecular cloud interface in M17SW, it is found that the models cannot reproduce the observed distribution of species. Scans of [CII] $158 \mu \mathrm{m}$ emission from M17SW $(1,2)$ show that the ionised carbon abundance decreases much less rapidly with distance from the cloud surface than expected for the cloud density derived from molecular line observations (3). To account for this it has been suggested that, at least to a depth of a few parsecs, the molecular cloud has a clumpy or fragmented structure, with the interclump material having a low density and a low FUV optical depth (1). Thus some of the FUV passes through the gaps between the clumps to ionise the surfaces of fragments deep within the cloud, so increasing the depth to which [CII] is detected.

\section{The Model.}

To simulate observations of an edge-on, fragmented PDR, a model is constructed from a spatially random distribution of cloud fragments. Each spherical clump consists of an optically thick cool dense core, and a relatively less dense warm envelope. For each clump, its probability of being illuminated by radiation from the ionising source, being in the shadow of foreground cores, or being prescreened by some amount of foreground envelope material, is calculated from the survival probability of a ray of light travelling from the FUV source to the centre of the clump. Having, calculated the distribution of conditions under which clumps exist, a one dimensional chemical model (4) is run for a representative population. This gives the average densities of say, $\mathrm{C}^{+}$, for each of the envelopes and cores. Using these values the column density along each line of sight is calculated. 
This is converted to an intensity via the transfer equation assuming a constant source function and using the relations of Crawford et al. (5) for the optical depth. This emission is then convolved with a gaussian beam pattern to simulate the $[\mathrm{CII}] 158 \mu \mathrm{m}$ observations.

\section{Results.}

Observations of the PDR in M17SW $(1,6)$ have indicated the presence of warm dense gas $\mathrm{T} \simeq 250 \mathrm{~K}$, and $\mathrm{n}_{H_{2}} \simeq 10^{4.5} \mathrm{~cm}^{-3}$, coexistent with cooler less dense material, $\mathrm{T} \simeq 50 \mathrm{~K}$, and $\mathrm{n}_{\mathrm{H}_{2}} \simeq 10^{5.5} \mathrm{~cm}^{-3}$.

For the FUV radiation to penetrate two or three parsecs into the cloud, and still cause ionisation of carbon on the surfaces of clumps there, then the density of the interclump medium must be less than $\mathrm{n}_{\mathrm{H}_{2}} \sim 500 \mathrm{~cm}^{-3}$, assuming it is not dust depleted. Many high resolution single dish and interferometer observations have revealed density enhancements of a few tenths of a parsec across, or less. These values are used for the standard clump density and temperature structure. With a clump diameter of $0.4 \mathrm{pc}$, an extent along the line of sight of $2 \mathrm{pc}$, and a volume filling factor of 0.2 , the model gives the intensity distribution as shown in Fig. 1. This corresponds to two to three clumps along a line of sight through the cloud.

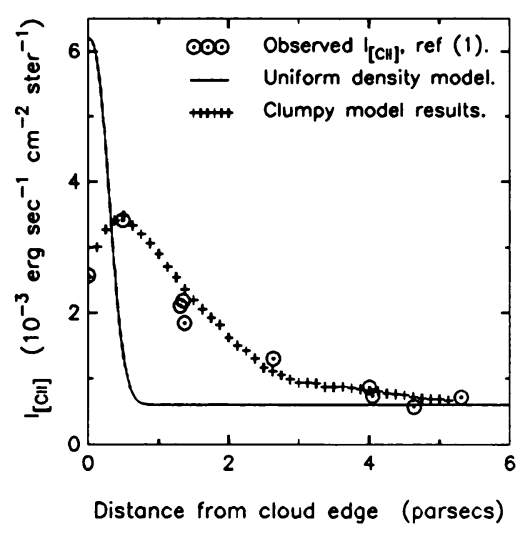

Figure 1. [CII] $158 \mu \mathrm{m}$ emission in M17SW.

The simulated $\mathrm{I}_{[C I I]}$ scan matches the observed one to within the accuracy of the observations. The [CII] intensity curve convolved with a $50^{\prime \prime}$ beam, predicted for a PDR of uniform density $\mathrm{n}_{\mathrm{H}_{2}}=10^{4.5} \mathrm{~cm}^{-3}$ is also plotted. The extended low intensity emission observed by Stutzki et al. (1) is supposedly due to embedded B-type stars, so one does not expect the model to reproduce this characteristic. Thus a constant $\mathrm{C}^{+}$column density corresponding to this emission has been added on to both model results before calculating the intensity. It is evident that the results of the clumpy model are a much better fit to the observations than the uniform density model.

\section{Conclusions.}

By using a model that attempts to mimic both the chemical and physical characteristics of a PDR, it has been possible to add weight to the interpretation of the observations in terms of a clumpy or fragmented PDR. If the M17SW PDR is not as closely edge on as has been thought, then this conclusion is more tentative. A more complicated interface geometry may produce a similar [CII] distribution without clumping. Currently work is underway to include observations of ${ }^{\prime} \mathrm{CO}$ and other molecular species to better constrain this model.

\section{References.}

(1) Stutzki, J., Stacey, G.J., Genzel, R., Harris, A.I., Jaffe, D.T., Lugten, J.B., 1988, Ap.J., 332, 379.

(2) Matsuhara, H., Takao, N., Shibai, H., Okuda, H., Mizutani, K., Maihara, T., Kobayashi, Y., Hiromoto,

N., Nishimura, T., and Low, F.J., 1989, Ap.J.Lett., 339, L67.

(3) Snell, R.L., Mundy, L.G., Goldsmith, P.F., Evans, N.J., Erickson, N.R., 1984, Ap.J., 276, 625.

(4) Monteiro, T.S., and Pinnock, S., 1990, in prep.

(5) Crawford, M.J., Genzel, R., Townes, C.H., and Watson, D.M., 1985, Ap.J., $291,755$.

(6) Harris, A.I., Stutzki, J., Genzel, R.,Lugten, J.B., Stacey, G.J., Jaffe, D.T., 1987, Ap.J.Lett., 322, L49 